developed, to investigate the associations between exposure and the presence of skin damage.

Method Based on ICNIRP publications, a detailed questionnaire, considering both working and leisure UV exposure, was developed and applied in group of volunteers and in patients affected by Non Melanoma Skin Cancers (NMSCs), to evaluate whole-life SR exposure and the relevant factors associated, including exposure habits, environmental factors and use of protections.

Results In patients with NMSCs, we found that the co-presence of in situ squamous cell carcinoma (SCC) and invasive NMSCs was more frequent in OWs than in indoor workers (IWs), as was also the frequency of multiple skin lesions. The prevalence of lesions in the most exposed body areas, as the face, was significantly higher in OWs, while working in shades was negatively associated with skin lesions. Also working posture was relevant: adopting a bent-over position was associated with lesions on the head. For leisure activities, the questionnaire application confirms the relevant role of tanning beds, in particular for body areas usually not highly exposed to SR, and the relevance of intense exposure in the middle hours of the vacation days ('sunbaths'), significantly associated with NMSCs presence.

Conclusion A detailed questionnaire-based evaluation in subjects with NMSCs can be very useful for estimating the whole-life individual UV exposure and the relevancy of occupation. Furthermore, an integration with personal dosimetric UV measurements can lead to a semi-quantitative evaluation, to set out exposure levels and different exposure modalities (e.g. intermittent vs cumulative) for various occupations and, possibly, for the different SCs.

\section{6c INTEGRATED CARE FOR WORKERS WITH OCCUPATIONAL SKIN CANCER IN THE GERMAN SOCIAL ACCIDENT INSURANCE SCHEME - REHABILITATION WITH ALL APPROPRIATE MEANS PROVIDED BY THE STATUTORY SOCIAL ACCIDENT INSURANCE IN GERMANY}

Bettina Lindemann. Institution for Statutory Accident Insurance and Prevention for Health and Welfare Services, Germany

\subsection{6/oemed-2018-ICOHabstracts.248}

Introduction Skin cancer caused by exposure to certain harmful substances has been a part of the list of occupational diseases in Germany for some time. In 2015 certain types of skin cancer (squamous cell carcinoma or multiple actinic keratosis of the skin caused by natural UV-irradiation) were added as a new occupational disease to the list of occupational diseases. These types of skin cancer are recognised as occupational disease if the insured person has been exposed to certain effective doses of sunlight at his or her workplace. There is a rising number of such cases reported to the statutory social accident insurance and its institutions which are the entities in charge of prevention, rehabilitation and compensation of occupational diseases in accordance with the German social security system. The institutions for statutory social accident insurance have developed concepts to handle the growing numbers of these cases.

Methods Based on research and the discussion with medical experts, standards for the procedure, for the medical treatment, the rehabilitation of workers affected by occupational skin cancer and the prevention of occupational skin cancer have been established. Concepts for follow-up care have been developed and new methods of therapy have been admitted for the medical treatment of patients suffering from occupational skin cancer.

Results The statutory social accident insurance has established measures to provide effective methods of rehabilitation for the patients concerned in accordance with the basic principle governing this social security scheme in Germany - acting 'with all appropriate means'.

Discussion Apart from providing effective help for the workers affected a major aim for the institutions of statutory accident insurance is the prevention of occupational skin cancer caused by exposure to natural UV-irradiation. The institutions of statutory social accident insurance will continue their efforts to reduce the risk for workers of contracting skin cancer caused by exposure to natural UV-irradiation at the workplace.

\section{$1616 \mathrm{~d}$ ON THE UV EXPOSURE OF WORKERS IN EUROPE IN GENERAL AND GERMANY IN PARTICULAR: WHAT DO WE KNOW? RESULTS OF THE GENESIS-UV PROJECT}

Marc Wittlich. Institute for Occupational Safety and Health (IFA), Sankt Augustin, Germany

\subsection{6/oemed-2018-ICOHabstracts.249}

Introduction We are exposed to solar ultraviolet radiation (UVR) every day, during work and leisure time. To date, there is only little knowledge about the level of irradiance. Already in 1992, UVR has been graded a group 1 carcinogen by the International Agency for Research on Cancer (IARC). In an ageing society, along with an ideal of a brown skin, non-melanoma skin cancer (NMSC) incidence rates rapidly increase.

Methods For risk assessment and deduction of protective measures, real measured data serve best. Regarding solar UVR exposure, long-term personal dosimetry measurements is required. With GENESIS-UV, we designed a suitable system. With only little impairment, each test persons measures autonomously. Data are transferred via mobile service or internet to a data server once a week automatically. GENESIS-UV has proven to be feasible in collaborations in the whole world without any changes in the system.

Results Since 2014, about 800 test persons delivered data points representing about 80000 days of measurement - in Germany. We derived the annual irradiance for more than 100 occupations, and far more occupational activities as well. Interestingly, the irradiance values for the occupations cover a very wide range (www.dguv.de/genesis). Going deeper into the occupational activities, prevention-relevant information could be obtained. Since 2016, measurements have been expanded at European level.

Discussion Outdoor workers have to be protected from UVR exposure. During working hours, their exposure can be up to 4.5 times the leisure dose. Our approach from occupations to occupational activities enables us to suggest very distinct protective measures, and drawing a full picture of workers exposure. Particularly, we showed that already short exposure times may lead to a notable hazard. Thus, the definition of an outdoor worker has to be reviewed.

The study with GENESIS-UV is by far the largest study on UV irradiance of workers worldwide. 